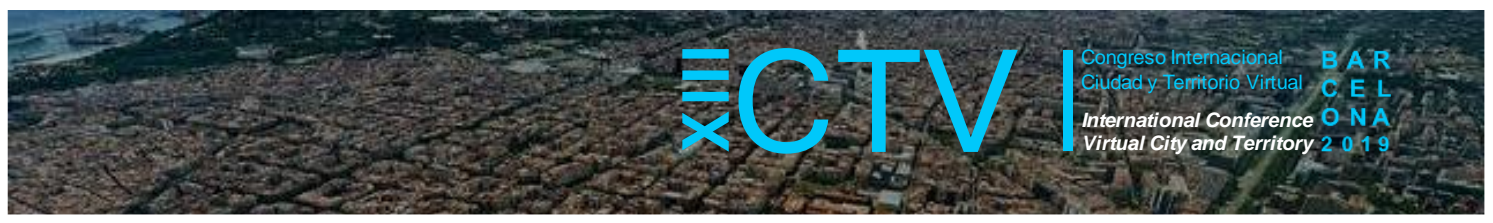

\title{
APROXIMACIÓN GRÁFICA A INDICADORES DE CALIDAD DE VIDA URBANA Y SOSTENIBILIDAD
}

\author{
Alonso-Montolio, Carlos ${ }^{1}$; Crespo, Isabel ${ }^{2}$; Coch, Helena ${ }^{3}$; Lopez-Besora, Judit ${ }^{4}$
}

Remisión inicial: 2019-06-16; Remisión definitiva: 2019-10-20; Publicación: 2019-12-21

Citación: Alonso-Montolio, C. et al. (2019). Aproximación gráfica a indicadores de calidad de vida urbana y sostenibilidad. En XIII CTV 2019 Proceedings: XIII International Conference on Virtual City and Territory: "Challenges and paradigms of the contemporary city": UPC, Barcelona, October 2-4, 2019. Barcelona:CPSV, 2019, p. 8615. E-ISSN2604-6512.DOI http://dx.doi.org/10.5821/ctv.8615

\begin{abstract}
Resumen
Las características del entorno urbano construido influyen en el comportamiento y en el bienestar de los usuarios. En las ciudades del entorno mediterráneo, con clima templado, las condiciones ambientales son favorables al bienestar del peatón, pero no siempre son suficientes ni homogéneas en todas las calles de un mismo distrito. Hay diferencias claramente perceptibles que hacen que un peatón describa rutas, en sus recorridos diarios, que no siempre son las más cortas o las más eficientes. Las herramientas de análisis urbano que aportan datos fiables y medibles de estas características se han convertido en un recurso útil para la comunidad científica de este ámbito y para los foros donde se centra la toma de decisiones sobre política urbana a pequeña o a gran escala. En este trabajo se ha tomado como base una de estas herramientas, el Transit Orientated Development Standard (TOD Standard), un instrumento de puntuación definido por el Institute for Transportation and Development Policy (ITDP), para evaluar un total de 12 áreas urbanas de las ciudades de Roma y Barcelona. El trabajo pone en evidencia que la representación gráfica de unos valores en un mapa aporta información complementaria muy importante y que no aparece en la simple cuantificación numérica. La representación de datos geográficamente localizada añade información contextual a los valores numéricos y, convenientemente ajustada en sus valores y escala, permite evaluar la calidad del espacio urbano a pequeña escala. Los criterios que fueron establecidos para tipologías urbanas propias de ciudades dispersas, cuando se han aplicado a ciudades con una morfología compacta requieren un ajuste a esa realidad y sugieren cualidades que no se ven en las tablas de valores. Se pone en evidencia que la representación en el mapa es la mejor manera de leer, sobre el plano, qué puede hacer que unas calles sean más transitadas que otras. Este trabajo demuestra que situar en el mapa los datos de puntuación del TOD completa la información y permite interpretar cualidades de la vida urbana fundamentales para fomentar la movilidad a pie. Tener en cuenta datos cualitativos, además de los cuantitativos, puede ser decisivo para asegurar el éxito de proyectos urbanos que tengan como objetivo fomentar los trayectos peatonales como sistema de movilidad prioritario.
\end{abstract}

\section{Abstract}

The characteristics of the built urban environment influence the behaviour and well-being of the users. In the Mediterranean cities, with mild climate, the environmental conditions are favourable to the well-being of the pedestrian. However, they are not always homogeneous in all the streets of the same district. There are perceptible differences that cause that pedestrians take some routes, in their daily journeys that are not always the shortest ones or the most efficient. Urban analysis tools that provide reliable and measurable data of these characteristics have become a useful resource for the scientific community in this area and for forums where decision-making is focused on urban policy on a small or large scale. In this work, one of these tools, the Transit Orientated Development Standard (TOD Standard), a scoring instrument defined by the Institute for Transportation and Development Policy (ITDP), has been taken as a basis for evaluating a total of 12 urban areas of the Cities of Rome and Barcelona. The work shows that the graphical representation of some values on a map provides very important complementary information that does not appear in the simple numerical quantification. The geographically located data representation adds contextual information to the numerical values. Conveniently adjusted in their values and scale, it allows evaluating the quality of the urban space on a small scale. When applying the criteria established for urban typologies typical of sprawl cities to cities with a compact morphology, an adjustment to that reality is required. They also suggest qualities that are not seen in the tables of

\footnotetext{
${ }^{1}$ Profesor Lector Serra-Hunter. AiEM. Dpto. Tecnología de la Arquitectura. ETSAB, UPC, https://orcid.org/0000-00030877-7212; ${ }^{2}$ AiEM Dpto. de Expresión Gráfica Arquitectónica. ETSAV, UPC, https://orcid.org/0000-0002-2422-0935; ${ }^{3}$ AiEM Dpto. de Tecnología de la Arquitectura. ETSAB, UPC, https://orcid.org/0000-0001-9524-8057; ${ }^{4}$ AiEM Dpto. de Tecnología de la Arquitectura. ETSAB, UPC, https://orcid.org/0000-0002-8659-9346 *Correo de contacto: carlos.alonsomontolio@upc.edu
} 


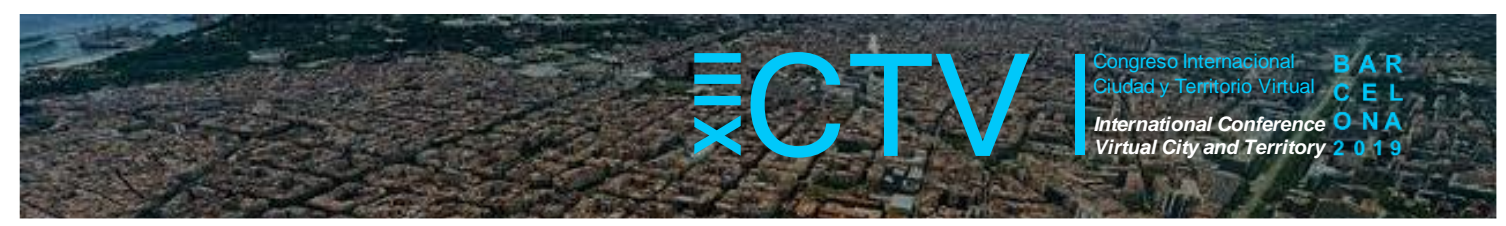

values. It is revealed that map representation is the best way to understand what can make some streets busier than other streets. This work demonstrates that placing the TOD scoring data on the map completes the information and allows interpreting qualities of urban life that are essential for promoting mobility on foot. Taking into account qualitative data, in addition to quantitative data, can be decisive to ensure the success of urban projects that aim to promote pedestrian paths as a priority mobility system.

Palabras Clave: Representación gráfica; Análisis urbano; Calidad urbana; TOD Standard

Key words: Graphical representation; Urban analysis; Livability; TOD Standard

\section{Introducción}

Los grandes movimientos migratorios desde las zonas rurales a las ciudades han reclamado la atención de la comunidad científica para profundizar en los estudios sobre la calidad de la vida urbana y para caracterizar la forma de la ciudad. Ante la perspectiva de continuar con un crecimiento extensivo de las ciudades, se ha acentuado el interés por promover la densificación de las conurbaciones para mitigar la invasión desbocada del territorio circundante a ellas. Junto a este repliegue del crecimiento, crece el consenso por reducir la contaminación ambiental provocada por la movilidad y el transporte con sistemas contaminantes.

Aunque el aprovechamiento de los recursos naturales y la reducción de los residuos generados en las ciudades son dos componentes imprescindibles a tener en cuenta para ajustar el impacto ambiental de las grandes concentraciones urbanas, hay otros aspectos, tan importantes como éstos, que también hay que considerar. Existe un consenso general entre la comunidad internacional según el cual el diseño de las ciudades debe tener en cuenta a sus usuarios y especialmente debe favorecer los desplazamientos peatonales, cosa que beneficiará la salud de las personas tanto por el ejercicio físico, como por una mejor calidad del aire que respiran (Southworth, 2005).

Pero las políticas que pretenden resolver problemas masivos, deben atender el detalle a escala individual para ser efectivos a escala general, especialmente cuando se trata de incidir en el comportamiento de las personas. La densificación urbana, como alternativa al crecimiento extensivo, puede tener repercusiones en la vida cotidiana que se han de saber entender, para aprovechar las ventajas y minimizar los posibles perjuicios de una intervención en las condiciones de calidad de vida urbana. Interesa tener instrumentos de soporte sobre los que basar la intervención en el medio urbano para mejorar las condiciones de vida de cada ciudadano.

\subsection{Ciudades para pasear}

Múltiples estudios ponen en valor las ventajas sociales, económicas, sobre la salud y sobre el medio ambiente de caminar como mejor medio de transporte para las distancias cortas (Dörrzapf, 2019). Puesto que los desplazamientos peatonales se caracterizan por ser el medio de transporte con más adherencia y aseguran una rica y cercana relación con el entorno (Mezoued, 2016), los rasgos del contexto urbano, donde han de tener lugar los trayectos a pie, se convierten en clave para que su promoción tenga éxito. Caminar es el método más natural de transporte para el ser humano y se ha desarrollado a lo largo de los siglos. Nuestro cuerpo, nuestra percepción sensorial y nuestra mente se identifican, y se ha configurado como son, gracias a este tipo de movilidad. Por tanto, es conveniente comprender qué valores son los que caracterizan la capacidad de un área urbana para hacerla paseable (Pozueta et al., 2009). 


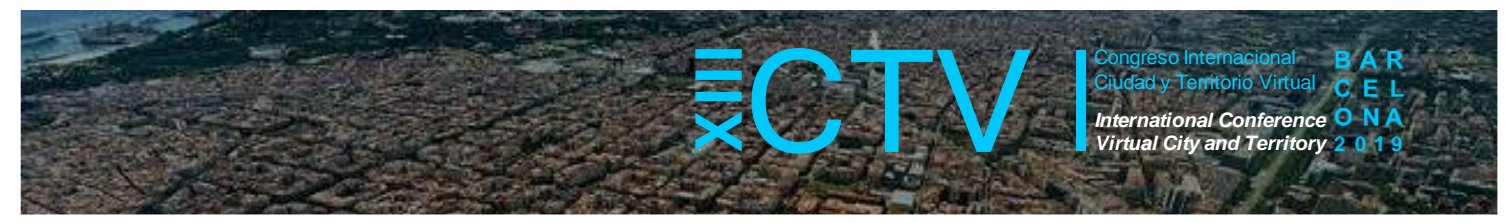

Pero incluso en ciudades que se pueden calificar globalmente como paseables hay zonas que disuaden del paseo a sus usuarios, por sus características particulares. Muchas veces los transeúntes escogen trayectos más largos, pero más agradables, más amables que otros más rápidos. La componente emocional es muy importante en los comportamientos de la movilidad de los usuarios del espacio público (Ma, 2014). Caminar puede llegar a ser difícil en ciertos ambientes (Kaufmann, 2011; Gehl, 2010). La representación en un mapa de esas características son las que pueden revelar esa localización para poder tomar decisiones 0 establecer políticas municipales de mejora en las ciudades, a escala de barrio.

En este trabajo se ha utilizado el Transit Orientated Development Standard (TOD Standard), desarrollado por el Institute for Transportation and Development Policy (ITDP) como herramienta capaz ayudar en la toma de decisiones relacionadas con el tránsito urbano mediante un sencillo sistema de puntuación. Tiene como objetivo maximizar los beneficios del transporte público y la movilidad no motorizada, teniendo en cuenta en primer lugar el beneficio de las personas que habitan en la ciudad.

El sistema de puntuación TOD es sencillo y ágil de aplicar, razón por la cual se ha considerado idóneo para este análisis. El sistema de puntuación TOD establece ocho principios: caminar, pedalear, conectar, transportar, mezclar, densificar, compactar y cambiar, definidos por catorce objetivos, que a su vez se evalúan mediante uno, dos o tres sistemas de medición. La puntuación final máxima es 100 (ITDP, 2017).

En este trabajo nos vamos a centrar en el principio "caminar" que da 15/100 puntos. Como caminar es la forma más saludable, barata y limpia para recorrer cortas distancias, se consideran factores clave para favorecer los recorridos a pie que sean: seguros, activos y cómodos. Estos serán los tres objetivos en que se centrará el principio "caminar": A) los dominios del peatón son seguros, completos y accesibles; B) el espacio del peatón es dinámico y animado; y finalmente C) el espacio del peatón es templado y confortable. En este estudio nos hemos centrado en el objetivo B. La actividad genera actividad. Andar es agradable cuando las aceras están pobladas y están animadas. Esto es más probable cuando hay servicios, comercios y actividades en las plantas bajas de los edificios. Los dos sistemas de medir este objetivo serán B.1.3) Fachadas visualmente activas y B.1.4) Fachadas físicamente permeables.

\subsection{Datos y mapas}

La representación gráfica de la información ha sido desde hace siglos una componente cada vez más reconocida en contextos científicos. Aunque el dibujo ha sido un modo de comunicación muy ágil para la humanidad desde sus orígenes, no siempre ha tenido el reconocimiento que merece como medio científico. Este trabajo viene a abundar en la idea de que las imágenes no sólo son atractivas visualmente, sino que aportan información que no puede hacerse evidente sólo con tablas de números. El mapa de Londres realizado por el médico John Snow en 1854, que le permitió relacionar el agua procedente de una fuente contaminada como el origen de la epidemia de cólera, puede considerarse uno de los primeros ejemplos de georreferenciación conocidos. Los gráficos de C. J. Minard para representar el avance, avatares y desastre de la Gran Armada durante la campaña de Rusia de Napoleón Bonaparte en 1812 también podrían considerarse pioneros, no solo en la visualización de datos, sino de algo más interesante aún como es el tratamiento dinámico de datos (Robinson, 


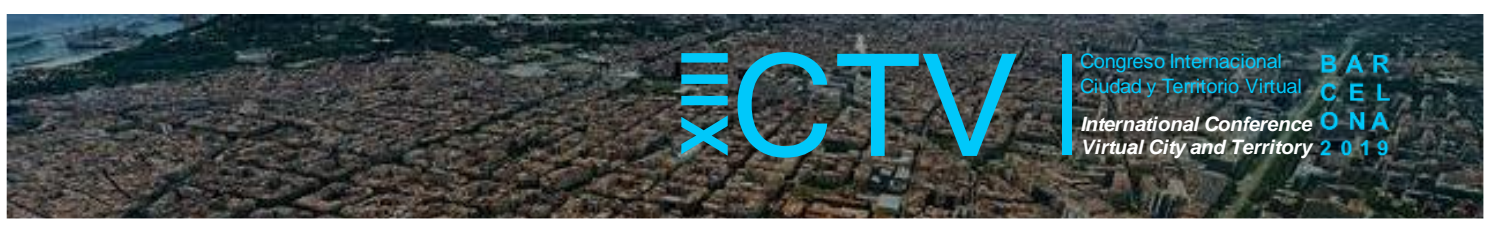

1967; Friendly 2002). Estos dos ejemplos, citados en numerosos escritos, son paradigmáticos como casos en los que el dibujo añade información a la mera consignación de los valores.

Las tecnologías actuales permiten gestionar gran cantidad de datos y representar gráficamente esta información en mapas extensos, a partir de los cuales se pueden hacer análisis muy detallados sobre diversos temas. Pero también podemos dar expresión gráfica a un número de datos mucho menor para hacer análisis detallados que, una vez establecidos o definidos, se pueden extrapolar a bases de datos masivas.

Los datos recogidos en el trabajo que aquí se presenta se han convertido en un mapa de información, asignándole a cada dato un código con el que representar su valor cuantitativo. La dificultad con la que nos enfrentamos está en saber seleccionar la información que conviene destacar de entre los datos disponibles y cómo hemos de representarla para hacer legibles los mapas. De este modo, se han puesto en evidencia cuáles son las relaciones espaciales además de cuáles son las cantidades de los parámetros escogidos. Pero, sobre todo, se han puesto en evidencia no sólo cuán grandes o pequeños son los valores, sino dónde están las singularidades, o lo que es lo mismo, cómo se distribuye la regularidad.

\section{Objetivo}

El objetivo de este análisis es doble. Por un lado, el uso de sistemas de representación que referencien valores numéricos a un lugar preciso y en un contexto para poner de relieve que los mismos datos son distintos según la situación geográfica que tengan, aunque sean cuantitativamente idénticos. Se trata de determinar el criterio gráfico que haga aflorar información de contexto urbano asociada a esa cualidad de fachada visualmente activa o de fachada físicamente permeable.

Por otro lado, una vez que se ha aplicado la herramienta de puntuación de los estándares, mencionados antes, se constata que la poca graduación de la escala de valores de los estándares escogidos queda superada por la localización física de esos valores. Es decir, si asignamos la puntuación establecida por el estándar del sistema TOD, que se ha descrito a partir de ciudades dispersas, a una ciudad compacta, todos los valores calificarían la muestra con los valores máximos. Esto haría poco interesante el análisis. Pero sabemos, por nuestra experiencia, que dos calles aparentemente iguales en cuanto a su forma y con el mismo clima, pueden percibirse distintas, con una posibilidad de adherencia muy diferente; y puede ser que los peatones escojan recorridos más largos a cambio de caminar por calles más atractivas. Si se encuentra una graduación adecuada se puede asignar que las fachadas de una calle sean más o menos animadas, o más o menos permeables que las de otra calle, aun sin ajustar esa graduación, ya que el mapa que representa los valores aporta la información que falta. Con la información correcta y su localización bien representada en el mapa, podríamos ser capaces de cuantificar lo aparentemente subjetivo.

El objetivo será, por lo tanto, validar si el sistema de puntuación establecido en el TOD sirve para apreciar los grados de cualificación de los frentes de calle más o menos activos y permeables. Es decir, saber si las diferencias de valor de estos parámetros, entre una calle y otra, en el mismo barrio, se pueden detectar en el plano. Esto permitiría identificar en el dibujo si hay alguna razón para que un peatón concreto decida alterar el trayecto para su paseo o 


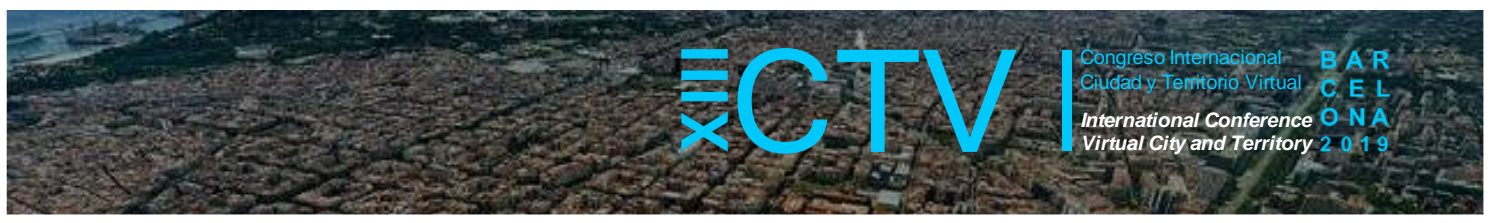

bien puede sugerir que hay alteraciones de la regularidad en la trama urbana dignas de considerar para potenciales intervenciones en el diseño del espacio público.

\section{Metodología}

Los datos con los que se han confeccionado los mapas han sido recabados de manera manual, recorriendo la calle y a partir de una inspección ocular, y no forman parte de ninguna base de datos automática.

Aunque la tecnología permite manejar una cantidad masiva de datos para confeccionar los mapas, el objetivo de este trabajo ha consistido en establecer el criterio gráfico y no tanto analizar los resultados sobre el fragmento de la ciudad escogido. Además, los datos de entrada que necesita el principio TOD escogido aquí no se corresponden con ningún dato disponible en las cartografías oficiales ni en las bases de datos a los que podemos tener acceso.

Las áreas de estudio seleccionadas para este caso son doce: tres de la ciudad de Roma (Giulio Agricola, Manzoni y Re di Roma) y 9 de la ciudad de Barcelona (la Barceloneta, el Clot, dos zonas de les Corts, Gràcia, tres zonas de Eixample y Sants).

Los barrios escogidos en las dos ciudades forman parte de tejidos urbanos de ciudad compacta, con densidades altas, la mayor parte de las manzanas tienen unas densidades netas (excluyendo la superficie del espacio público) de entre 300 y 900 habitantes por hectárea. Esta amplitud de valores se debe a que algunas manzanas incluyen fincas no habitadas, pero sí construidas, como son escuelas u hospitales, y otras manzanas son de edificios de vivienda en altura.

Los barrios analizados en Roma son fragmentos de ciudad de un radio de unos 500 metros mientras que en Barcelona se analizaron fragmentos del tejido urbano inscritos en un círculo de unos 200 metros de radio.

Esta diferencia no tiene ninguna trascendencia en los resultados. El análisis no pretendía llegar a un diagnóstico de las condiciones de los barrios, sino que pretendía validar que los parámetros son los adecuados para entender ciertas características de la calle y qué criterio gráfico es adecuado.

Los indicadores del TOD escogidos que se han mencionado antes son B.1.3. y B.1.4., referidos a las plantas bajas de las manzanas. Sobre los frentes de calle se han identificado los fragmentos con conexión visual a las actividades del interior de los edificios y se han identificado las entradas peatonales a los edificios que son los que recoge la figura siguiente:

Figura 1. Extracto de la tabla de puntuación del TOD

\begin{tabular}{|c|c|c|c|c|c|}
\hline \multirow{5}{*}{ WALK (15p.) } & \multirow{2}{*}{ A } & \multirow{2}{*}{ The pedestrian realm issafe and complete } & 1.1 & Walkways & 3 \\
\hline & & & 1.2 & Crosswalks & 3 \\
\hline & \multirow{2}{*}{ B } & \multirow{2}{*}{ Thepedestrian realm is active and vibrant } & 1.3 & Visually Active Frontage & 6 \\
\hline & & & 1.4 & Physically Permeable Frontage & 2 \\
\hline & C & The pedestrian realm istemperate and comfortable & 1.5 & Shade \& Shelter & 1 \\
\hline
\end{tabular}

Fuente: TOD Standard v2.1 


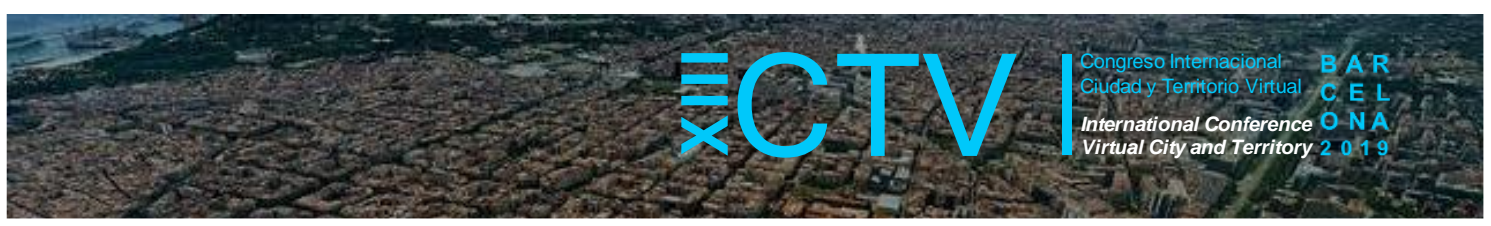

El primer indicador, Fachada Visualmente Activa (B.1.3.), mide las posibilidades de una conexión visual entre la acera y el interior de las plantas bajas y define el porcentaje de segmentos de la fachada en planta baja que tienen conexión visual con la actividad interior de los edificios (ITDP, 2017).

El método de medida calcula el porcentaje de tramos con conexión visual respecto del total del frente de fachada. Un valor de $20 \%$ se considera Visualmente activo. Este indicador computa hasta 6 puntos que se aportan al total del sistema de puntuación TOD, que es de 100 puntos.

Las mediciones de los frentes visualmente activos se han calculado sobre fotografías frontales de cada frente de calle. Para determinar este indicador, se ha cuantificado la proporción de fragmentos visualmente activos respecto del total.

El Segundo indicador, (B.1.4.) Fachadas Físicamente Permeables, se define por el número medio de tiendas, entradas a edificios y otros accesos peatonales por cada 100 metros de frente de manzana (ITDP, 2017). Se han contabilizado el número de entradas de este tipo en cada tramo de calle. Esta contabilización de puertas de acceso peatonal también se ha hecho in situ, por inspección ocular.

Figura 2. Frente de la calle Comte Borrell del distrito de les Corts de Barcelona

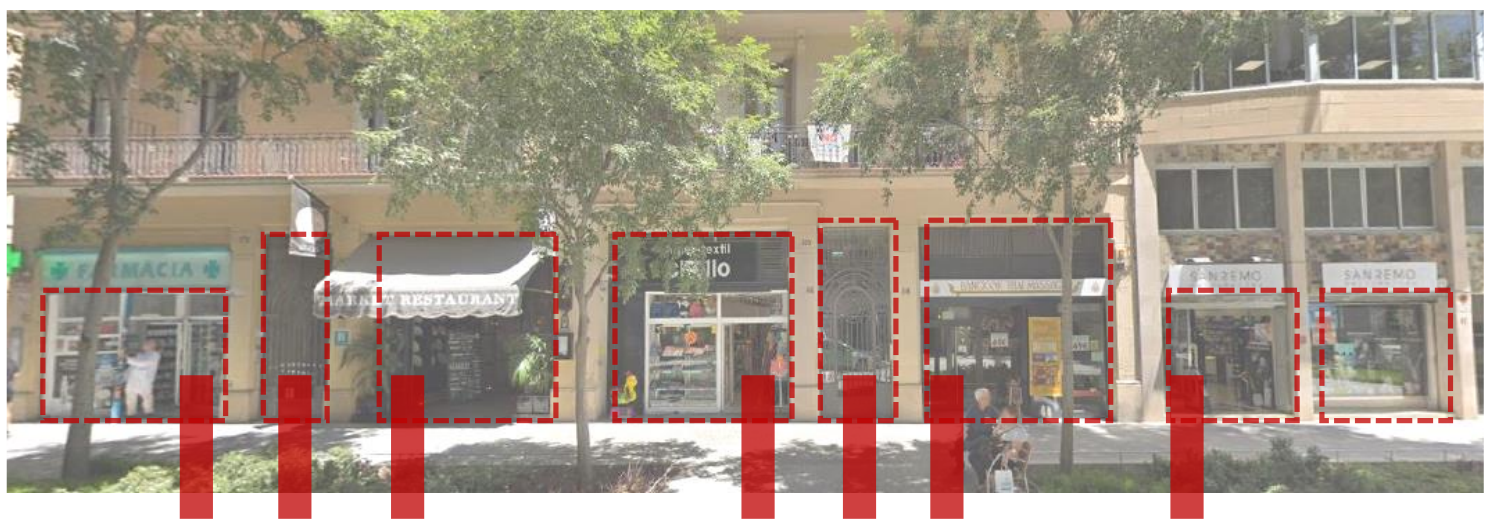

Nota: Los rectángulos rallados corresponden a las fachadas visualmente activas y los rectángulos sólidos a los accesos peatonales. Fuente: elaboración del autor

Con estos valores se han confeccionado los mapas del sector analizado y se ha definido una simbología adecuada para interpretar los resultados una vez obtenidos.

Para el indicador de Fachada Visualmente Activa se ha establecido una banda sobrepuesta en el perímetro de cada manzana, con una escala de 6 colores, según la cual los diferentes rangos del porcentaje se pueden distinguir a simple vista, como se detalla en la figura.

Para el indicador de Fachada Físicamente Permeable el criterio gráfico ha consistido en grafiar cada una de las entradas posibles detectadas sobre una banda sobre en el perímetro de cada frente de manzana. Se ha dibujado una escala de 3 colores que corresponden a tres horquillas de valores: de 0 a 3 entradas cada 100 metros de fachada, entre 3 y 5 cada 100 metros y más de 5 cada 100 metros. 


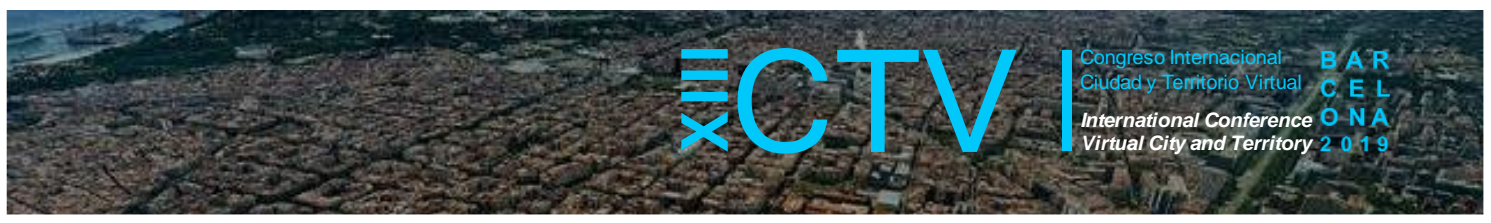

Figura 3. Mapa de Fachada Visualmente Activa en un fragmento de El Ensache de Barcelona

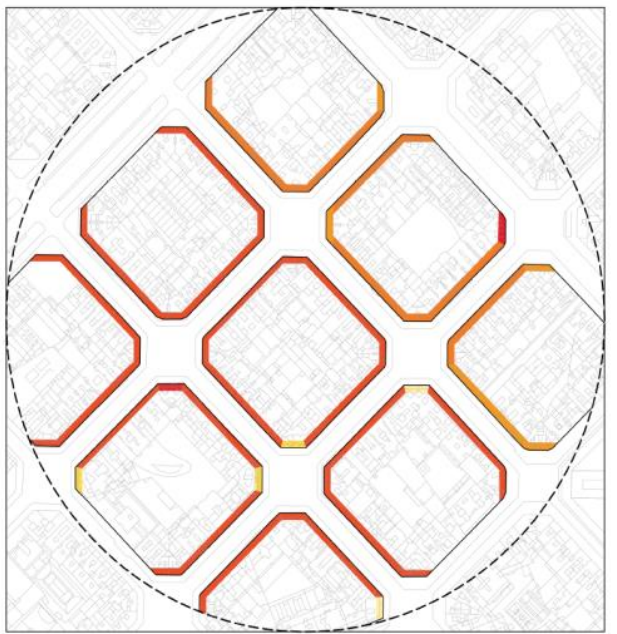

$$
\begin{aligned}
& \text { Less than } 50 \% \\
& 50 \%-60 \% \text { (2 points) } \\
& 60 \%-70 \% \text { (3 points) } \\
& 70 \%-80 \% \text { (4 points) } \\
& 80 \%-90 \% \text { (5 points) } \\
& 90 \%-100 \% \text { (6 points) }
\end{aligned}
$$

Fuente: elaboración del autor

Figura 4. Mapa de Fachada Físicamente Permeable en un fragmento de El Ensache de Barcelona
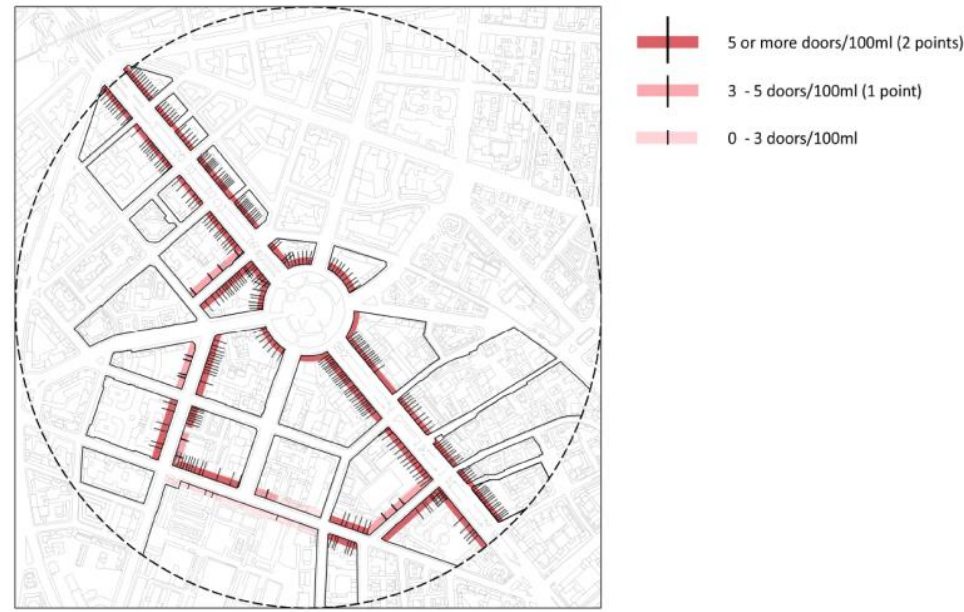

$1 \quad 0.3$ doors/100m

Fuente: elaboración del autor

\section{Casos de estudio}

Los doce barrios sobre los que se ha recogido la información tienen características urbanísticas similares entre ellos, en cuanto a densidades, tipología edificatoria y de usos, así como en que tienen una fuerte conexión con su entorno urbano inmediato. En todos los casos el uso urbanístico principal es de vivienda alternado con equipamientos propios de la ciudad compacta (escuelas, hospitales, centros culturales); los espacios viarios más abiertos corresponden a plazas o fragmentos de parques urbanos y el sistema viario sirve de soporte al tráfico rodado en calles con anchuras muy variables. No son calles peatonales ni centros históricos, donde la actividad turística podría condicionar la información de la vida ciudadana. En todos los casos se trata, pues, de zonas con una actividad cotidiana viva, propia de las ciudades europeas de esta latitud. La actividad comercial está muy centrada en el comercio de proximidad, el perfil social de la población corresponde a la sociedad media de la ciudad, tanto en la estructura de la pirámide de edad como en el reparto de género. 
Figura 5. Localización de los barrios estudiados en las dos ciudades
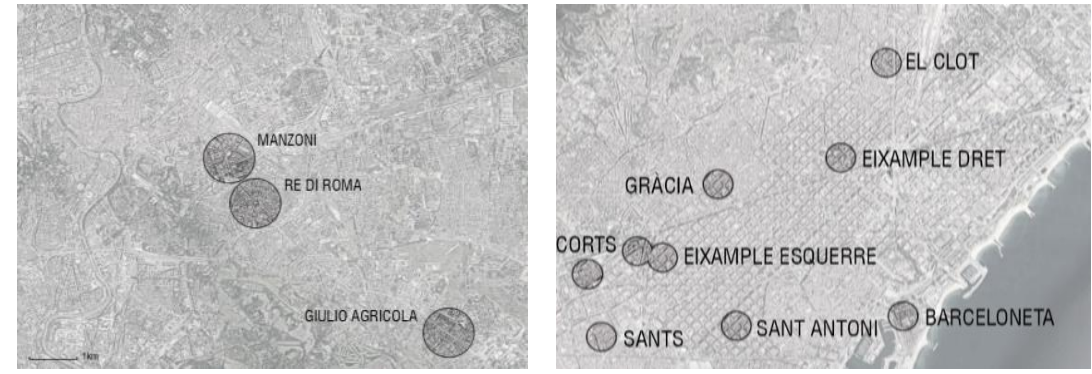

Fuente: elaboración del autor

En cuanto a la morfología urbana, se han estudiado barrios que tienen tramas muy diversas, unos con una gran regularidad formal en el trazado de sus calles, la altura de los edificios y en el tamaño de sus manzanas, como en el caso del Ensanche o de la Barceloneta, en Barcelona; y otros barrios con un tramado irregular como la zona de Re di Roma o Manzoni, en Roma.

Figura 6. Algunos de los fragmentos estudiados. De izquierda a derecha: Ensanche, Barceloneta, Re di Roma y Manzoni
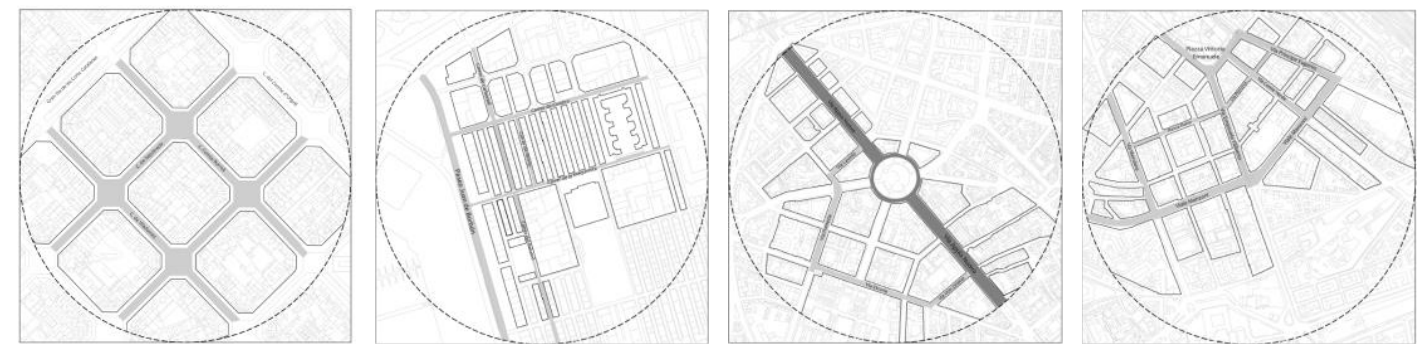

Fuente: elaboración del autor

A partir de los datos y una vez establecido el criterio gráfico para confeccionar los mapas se han elaborado los dibujos que representan los dos indicadores. La figura 7 muestra, de cuatro de los sectores estudiados, los tres dibujos que se han elaborado.

Figura 7. Mapas elaborados. Por columnas: vías estudiadas, Fachada Visualmente Activa y Fachada Físicamente Permeable. Por filas: Barceloneta, Ensanche, Re di Roma y Manzoni.
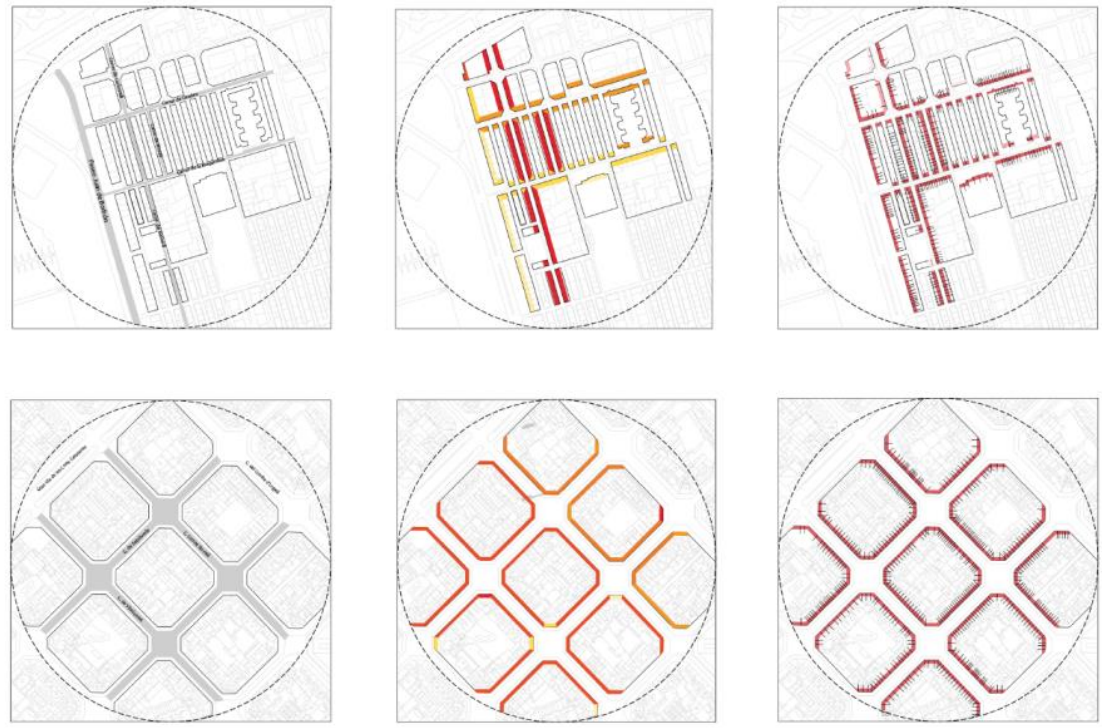

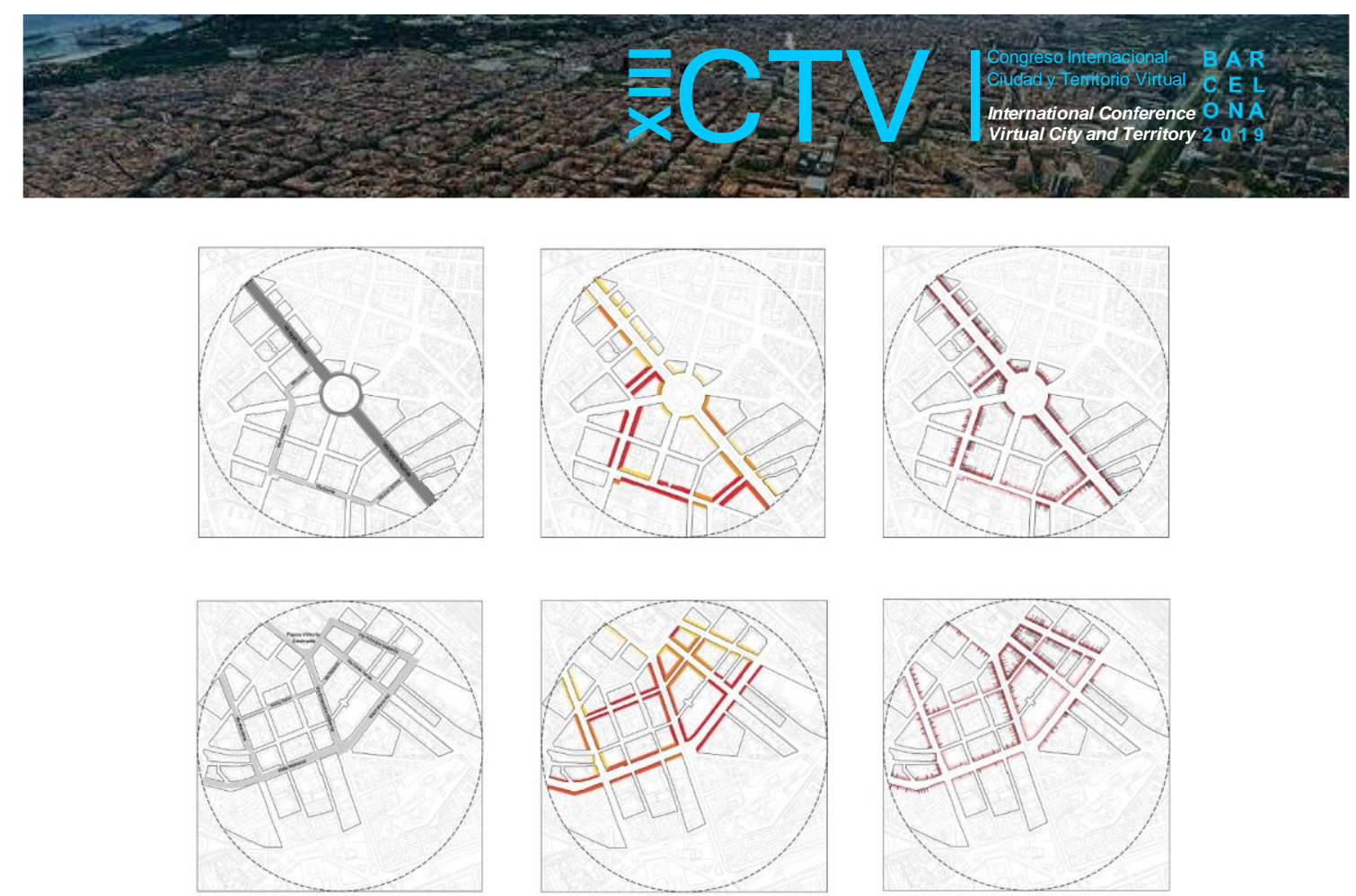

Fuente: elaboración del autor

A la izquierda el plano viario donde se destacan las vías de las que se pudo recoger información. En el centro el mapa que representa los diferentes grados de Fachadas Visualmente Activas y a la derecha de la fila, los mapas que representan las Fachadas Físicamente Permeables.

\section{Resultados y discusión}

Los datos obtenidos permiten cuantificar los indicadores descritos en el principio elegido del estándar del TOD y asignar una cualificación a cada tramo. Según el sistema TOD, a partir de los valores obtenidos se puede puntuar los barrios en conjunto, según la siguiente tabla:

\begin{tabular}{|c|c|c|c|c|c|}
\hline & \multirow[t]{2}{*}{ BARRIO } & \multicolumn{2}{|c|}{$\begin{array}{l}\text { FACHADA VISUALMENTE } \\
\text { ACTIVA }\end{array}$} & \multicolumn{2}{|c|}{$\begin{array}{c}\text { FACHADA FISICAMENTE } \\
\text { PERMEABLE }\end{array}$} \\
\hline & & $(\%)$ & POINTS & (entrances/100m) & POINTS \\
\hline \multirow{10}{*}{ 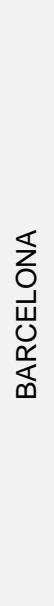 } & Eixample 1 & 46 & $0 / 6$ & 19 & $2 / 2$ \\
\hline & Eixample 2 & 54 & $2 / 6$ & 18 & $2 / 2$ \\
\hline & Eixample 3 & 50 & $2 / 6$ & 20 & $2 / 2$ \\
\hline & El Clot & 30 & $0 / 6$ & 18 & $2 / 2$ \\
\hline & Gracia & 45 & $0 / 6$ & 21 & $2 / 2$ \\
\hline & Les Corts 1 & 60 & $3 / 6$ & 12 & $2 / 2$ \\
\hline & Les Corts 2 & 36 & $0 / 6$ & 16 & $2 / 2$ \\
\hline & Barceloneta & 36 & $0 / 6$ & 13 & $2 / 2$ \\
\hline & Sants & 60 & $3 / 6$ & 11 & $2 / 2$ \\
\hline & Media de Barcelona & 46 & $1,1 / 6$ & 16 & $2 / 2$ \\
\hline
\end{tabular}




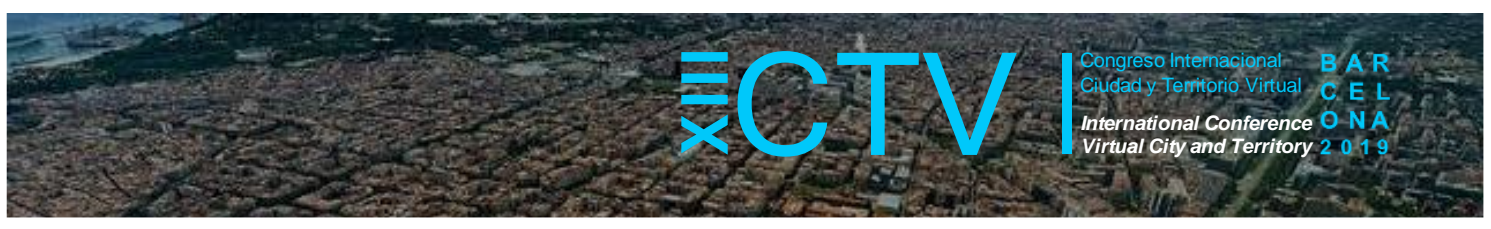

\begin{tabular}{llllll}
\hline Manzoni & 30 & $0 / 6$ & 6 & $2 / 2$ \\
\cline { 2 - 6 } & Re di Roma & 46 & $0 / 6$ & 11 & $2 / 2$ \\
\cline { 2 - 5 } & Giulio Agricola & 53 & $2 / 6$ & 11 & $2 / 2$ \\
\hline Media de Roma & 43 & $0,7 / 6$ & 9 & $2 / 2$ \\
\hline
\end{tabular}

Fuente: elaboración del autor

A partir de la tabla, bajo los criterios del sistema TOD, se concluye que hay una gran homogeneidad en la permeabilidad física de los frentes de calle en todos los barrios. O que son, en general, visualmente poco activos, puesto que los valores de este parámetro son raramente mayores de 3 sobre un máximo de 6.

Sin embargo, la representación de esos valores localizados en el plano arroja otro tipo de información. Si comparamos dos casos entre ellos, el Ensanche y la Barceloneta, ambos morfológicamente regulares, se advierte que la permeabilidad y la actividad visual se distribuyen de manera muy diferente (ver fig.7). Mientras que en el Ensanche se mantiene la regularidad -todos los frentes tienen la misma simbología y coinciden la permeabilidad i la actividad visual en esa uniformidad-; en la Barceloneta, hay una notable diferencia entre las calles interiores del barrio, longitudinales a las manzanas, y las calles trasversales a éstas. En las primeras hay un gran número de puertas, son físicamente muy permeables, pero hay una actividad visual muy baja, y en las segundas en cambio destaca su actividad visual y es en ellas donde se concentran los valores más altos del barrio.

Sin entrar a buscar las causas de cada situación, lo que interesa en este trabajo es que estos matices se detectan cuando se representan los valores en el mapa. El mapeado de la información define mejor las condiciones de la cualidad de unas condiciones ambientales que los números en una tabla.

La permeabilidad o el dinamismo pueden variar mucho entre un tramo y otro en un mismo sector y esto se puede apreciar gracias a la localización de los frentes visualmente activos o al número y secuencia de puertas de un tramo de una calle.

La figura 8 corresponde al indicador de Fachada Visualmente Activa, es decir, los porcentajes de segmentos transparentes respecto del total de la calle, en un fragmento del barrio de Les Corts de Barcelona. Este plano pone en evidencia que hay una gran concentración de frentes activos en algunos tramos de calle y que hay otros tramos mucho más opacos.

En la figura 9 el indicador Fachada Físicamente Permeable, es decir, el número de entradas en cada frente de calle, tiene una distribución muy irregular en todo el fragmento representado. Las calles son de dimensiones parecidas y la trama viaria es regular.

Si pensamos en un peatón que quiera atravesar el barrio, desde la esquina de la calle Anglesola con la calle Numancia hasta otro situado en la diagonal sur del plano, en la esquina de la calle Galileo con la Travesera de Les Corts, se pueden observar muchas combinaciones de recorridos con características muy diferentes. 


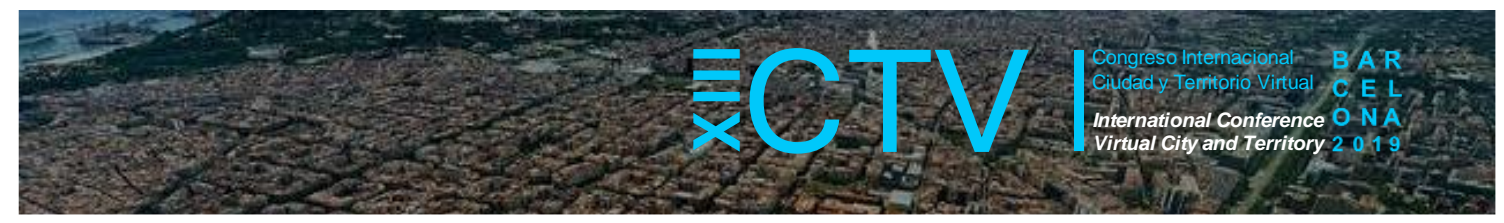

Un recorrido $u$ otro tendrán diferente cualidad en cuanto a permeabilidad y actividad visual. Hay recorridos que pueden llegar a pasar por frentes muy opacos y otros recorridos muy permeables y visualmente muy activos.

Figura 8. Mapa de Fachadas Visualmente Activas en el barrio de les Corts en Barcelona. En color verde un recorrido visualmente poco activo y en naranja un recorrido visualmente muy activo.

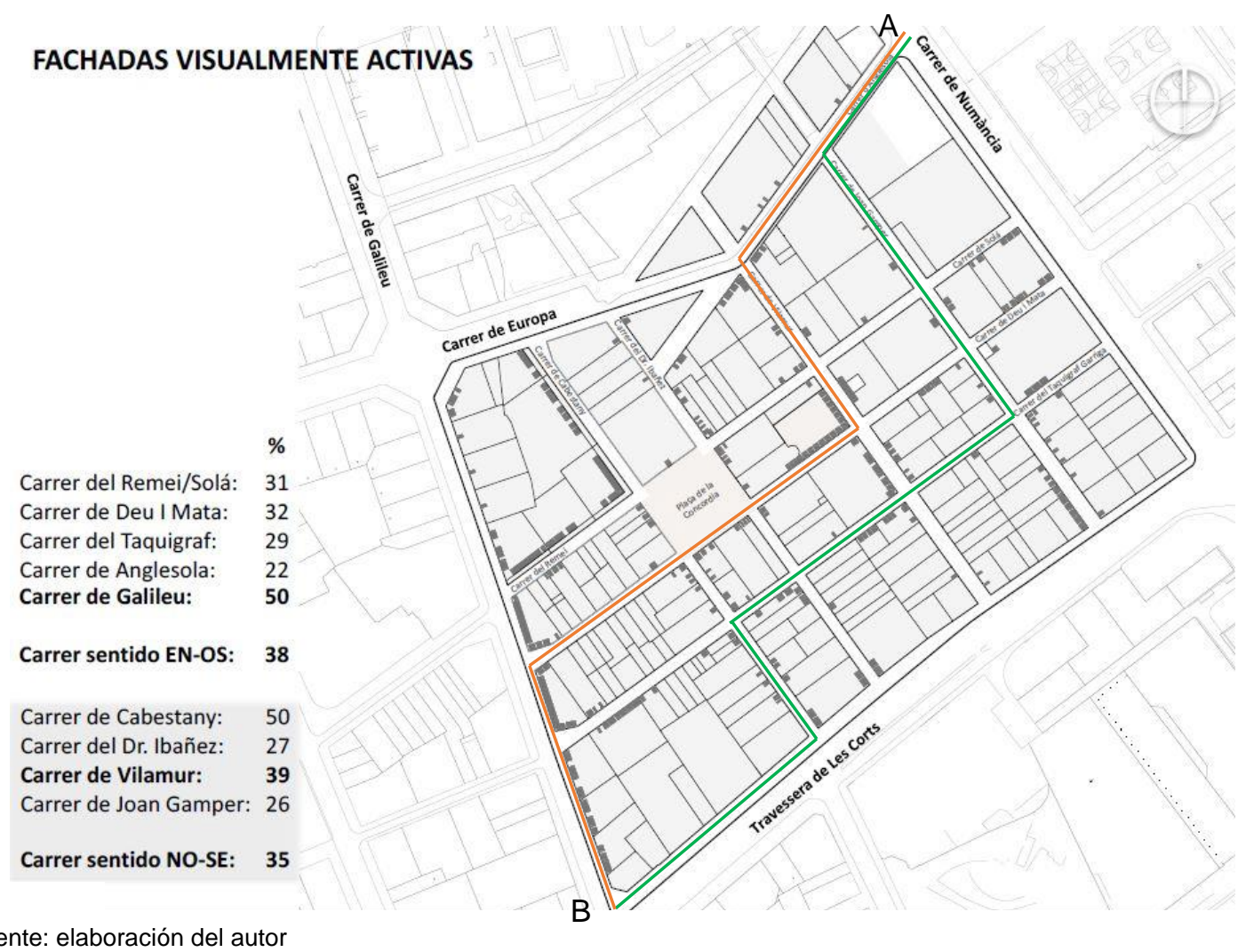

Fuente: elaboración del autor

En el mapa de Fachadas Visualmente Activas se ha dibujado un recorrido posible de color verde y otro de color naranja, con longitudes muy parecidas. El primero pasa por frentes de calle muy opacos y el segundo pasa por calles con muchas más ocasiones de mirar al interior de los edificios.

Para los mismos trayectos sobrepuestos en el segundo mapa, de Fachadas Físicamente Permeables, las características cambian: el recorrido verde permite pasar por calles con muchas más posibilidades de encontrarse con personas entrando y saliendo de los edificios que el recorrido naranja.

Caminar es un sistema de movilidad (Dörrzapf, 2019) con muchos beneficios sobre la vida de las personas, uno de los cuales es la autonomía que otorga al ciudadano. Se tiene más libertad de movimientos, menos dependencia de otros ciudadanos y de los servicios públicos, por lo que es uno de los sistemas más inclusivos. Otra característica es la capacidad de adherencia a lo largo del recorrido. Caminar genera una relación con la ciudad de contacto muy directo. Será importante, pues, favorecer que las características del espacio público permitan que esa adherencia se produzca, puesto que la posibilidad de que exista fomentará los trayectos a pie. El peatón es el que más se puede relacionar con el entorno inmediato, pero esa posibilidad de 


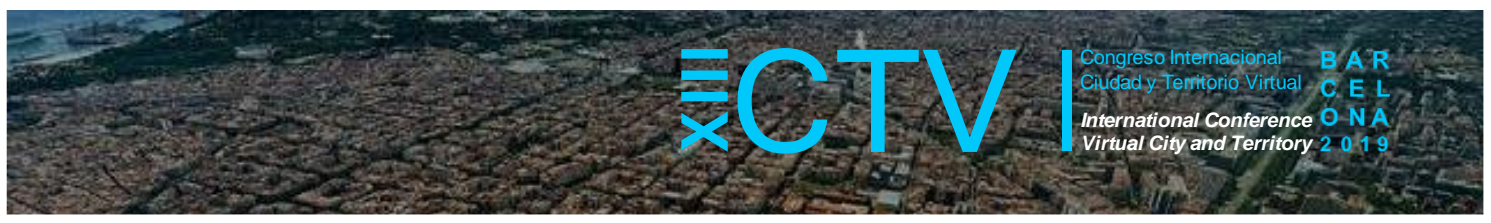

relación ha de ser recíproca. Los frentes urbanos edificados que tienen muchos accesos a los edificios, o con muchas ventanas que conecten visualmente el interior con el exterior, ofrecen un recorrido más seguro y amable. En cambio, calles poco permeables o visualmente poco activas disuaden de ir andando.

Figura 9. Mapa de Fachadas Físicamente Permeables en el barrio de les Corts en Barcelona. En color verde un recorrido físicamente muy permeable y en naranja un recorrido poco permeable

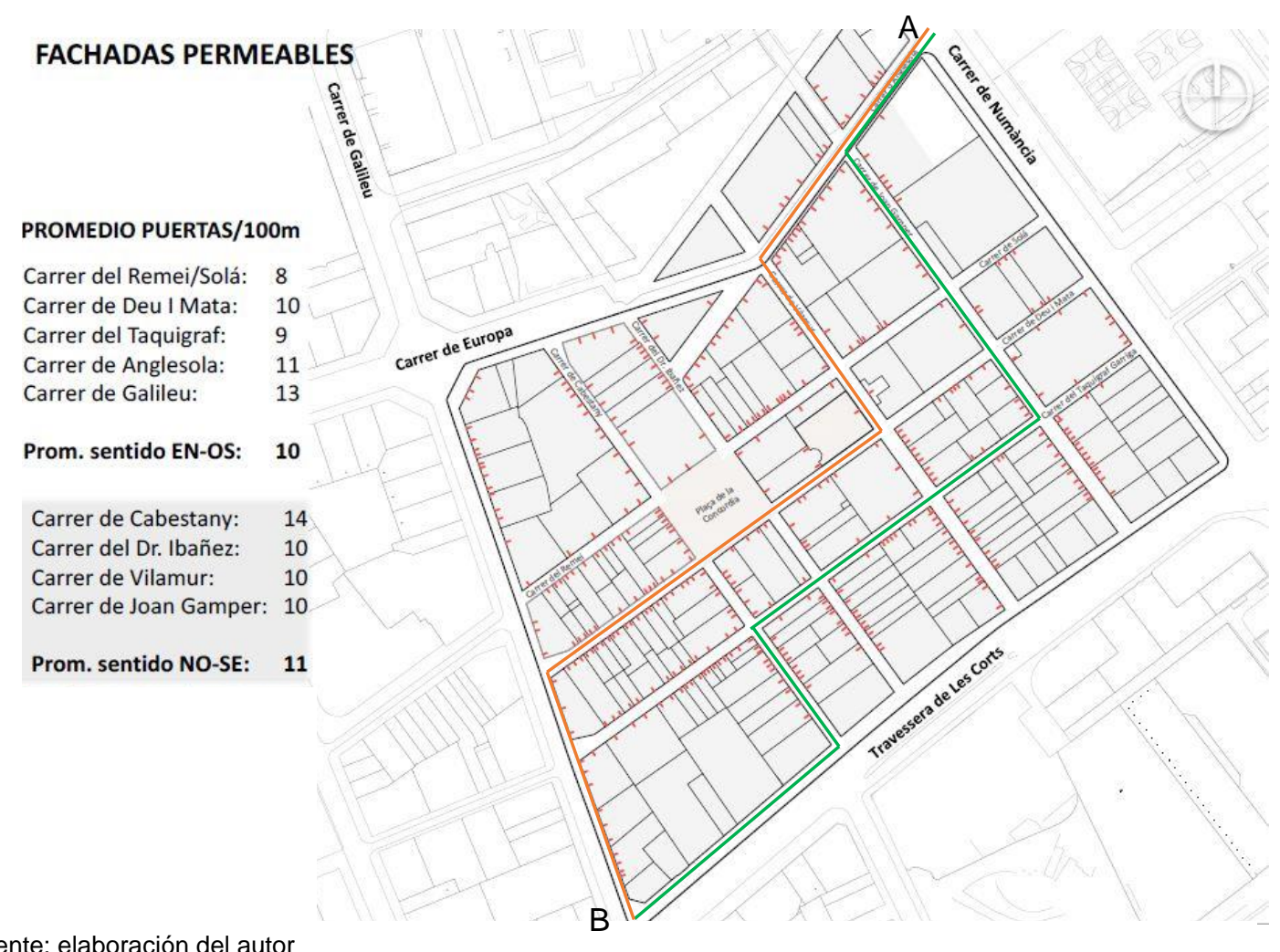

Fuente: elaboración del autor

Este caso de estudio, más exhaustivo, pone de manifiesto que no tiene por qué haber correspondencia ente un estándar y el otro. En la zona estudiada se aprecia que los frentes más activos visualmente no son los más físicamente permeables. Los frentes de fachadas con más puertas son unos y los frentes de fachadas más activos visualmente son otros. Aunque de la puntuación no se deduce este aspecto.

\section{Conclusiones}

Los indicadores como la actividad visual o la permeabilidad física de los frentes de fachada de calle son parámetros difíciles de cuantificar, aunque los estándares del TOD han intentado una aproximación que hemos considerado suficientemente válida.

La escala de los estándares que se estableció para modelos de ciudades dispersas no es la más adecuada para ciudades mediterráneas con una morfología urbana compacta y consolidada, pero la representación gráfica en un mapa de los valores, con unos códigos adecuados, diluye ese desajuste y aporta la información sin la cual las puntuaciones estándar quedan incompletas. 


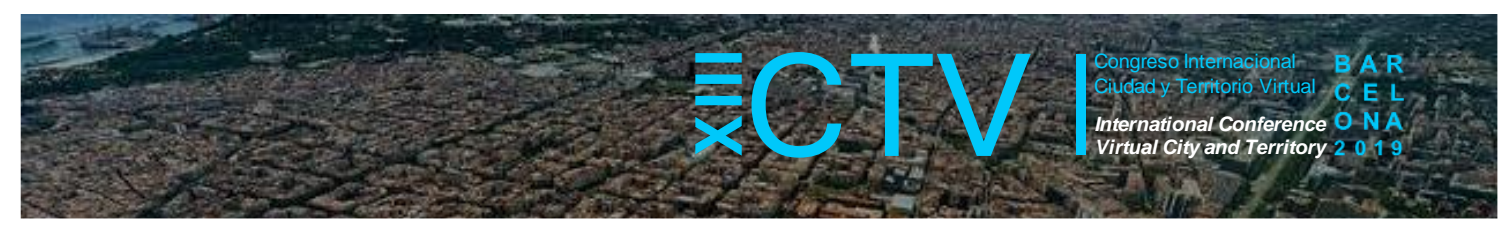

Este trabajo demuestra que representar esos valores en el mapa sin ninguna alteración métrica multiplica su eficacia y su utilidad. En ciudades muy consolidadas, en climas y culturas que favorecen los trayectos peatonales pero que las condiciones socioeconómicas o ambientales podrían disuadir a sus habitantes de ir andando, debemos saber medir qué condiciones pueden fomentar la movilidad peatonal para poden ahondar en esta política. El método utilizado muestra que se puede medir con muchos más matices la calidad de vida en el espacio público, si combinamos información y mapa.

La capacidad de adherencia que tienen los trayectos a pie deben encontrar la réplica en las características de los frentes de calle para que esa adherencia potencial se produzca y los mapas permiten analizar la realidad mejor que los datos.

Tener en cuenta datos cualitativos, además de los cuantitativos, puede ser decisivo para asegurar el éxito de proyectos urbanos que tengan como objetivo fomentar los trayectos peatonales como sistema de movilidad prioritario.

El fomento del paseo en las ciudades compactas europeas, de clima mediterráneo, puede apoyarse en estudios como este para saber cuantificar parámetros difíciles de medir, pero que muchas veces son los que aportan calidad a la vida urbana.

Agradecimientos: Este trabajo se ha desarrollado en el marco del proyecto de investigación BIA2016-77675-R financiado por el Gobierno de España. En la toma de datos han participado los estudiantes del MBArch de la línea AiE (UPC) del curso 18-19, así como los participantes al 5th International Workshop on Mediterranean Urban Morphology llevado a cabo en SOS Urban Lab (Sapienza Università di Roma) en mayo de 2019.

Contribución de los autores: Conceptualización: CAM, IC, HC y JL; Metodología: CAM, IC, HC y JL; Interpretación y análisis de resultados: CAM, IC, HC y JL; Escrito, revisión y edición: CAM, IC, HC y JL.

Conflictos de intereses: los autores declaran no tener conflictos de intereses.

\section{Bibliografía}

Arellano, B., \& Roca, J. (2010). El urban sprawl, ¿un fenómeno de alcance planetario? Los ejemplos de México y España. ACE: Architecture, City and Environment, 4 (12). DOI: http://dx.doi.org/10.5821/ace.v4i12.2489.

Dörrzapf L., Kovács-Győri A., Resch B. \& Zeile P. (2019). Defining and assessing walkability: an concept for an integrated approach using surveys, biosensors and geospatial analysis. Urban Development Issues, vol. 62, pp. 5-15.

Friendly, M. (2002). Visions and re-visions of Charles Joseph Minard. Journal ofEducational and Behavioral Statistics, 27(1), 31-51. http://dx.doi.org/10.3102/10769986027001031.

Gehl, J. (2010). Cities for People. Washington DC: Island Press. 


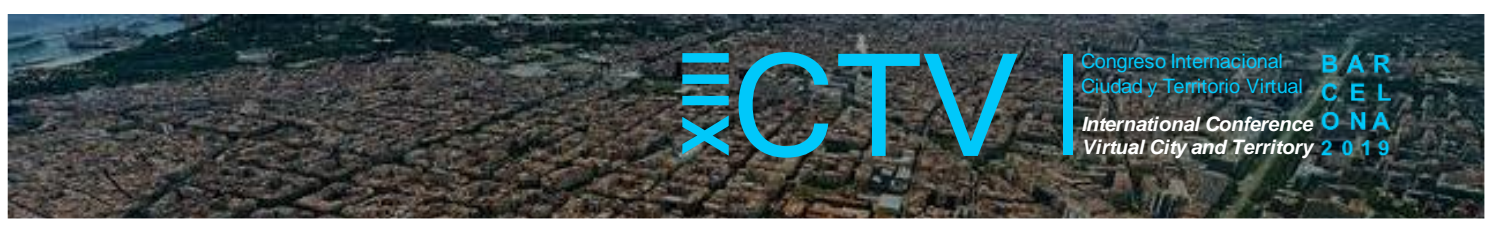

Gehl, J. (2011). Life between buildings: using public space. Washington DC: Island Press.

Gehl, J. \& Svarre, B. (2013). How to Study Public Life. Island Press, Washington DC. DOI: https://doi.org/10.5822/978-1-61091-525-0.

ITDP (2017). TOD Standard 3rd ed. ITDP (Institute for Transportation and Development Policy). New York. https://www.itdp.org/tod-standard/

Jacobs, J. (1961). The Death and life of great American cities. New York, USA: Random House.

Kaufmann, V. (2011). The individual motilities that make the city. Rethinking the city:Urban dynamics and mobility (pp. 65-84). Lausanne, Switzerland: EPFL Press.

Ma, L. (2014). The Objective vs. the Perceived Environment: What Matters for Active Travel. Dissertations and Theses. Paper 2090. Portland State University.

Mezoued, A. (2016). L'in-adhérence spatiale du tramway d'Alger à l'échelle locale. In M. Srir (Ed.). Dynamiques urbaines à Alger: La (re)fabrication de la ville en questions (pp. 247-265). Paris, France: L'Harmattan.

Montgomery, C. (2013). Happy city: Transforming our lives through urban design. New York, USA: Farrar, Straus and Giroux.

Pozueta Echavarri, J., Lamíquiz Daudén, F. J., \& Porto Schettino, M. (2009). La ciudad paseabe: recomendaciones para la consideración de los peatones en el planeamiento, el diseño urbano y la arquitectura. Madrid, Spain: Centro de Estudios y Experimentación de Obras Públicas.

Radovic, D. (Ed.). (2013). Measuring the non-measurable Workbook 1: Intensities in ten cities. Fujisawa, Japan: IKI (International Keio Institute) + Flick Studio.

Robinson, A. H. (1967). The thematic maps of Charles Joseph Minard. Imago Mundi: The International Journal for the History of Cartography, 21(1), 95-108. http://dx.doi.org/10.1080/03085696708592302.

Serra-Coch, G., Chastel, C., Campos, S., \& Coch, H. (2018). Graphical approach to assess urban quality: Mapping walkability based on the TOD-standard. Cities, 76 (January), 58-71. https://doi.org/10.1016/j.cities.2018.01.007.

Southworth, M. (2005). Designing the walkable city. Journal of Urban Planning and Development, 131(4), 246-257. https://doi.org/10.1061/(ASCE)0733-9488(2005)131:4(246) 